Pacific Journal of Mathematics

QUOTIENTS OF COMPLETE GRAPHS: REVISITING TH 


\title{
QUOTIENTS OF COMPLETE GRAPHS: REVISITING THE HEAWOOD MAP-COLORING PROBLEM
}

\author{
Jonathon L. Gross and Thomas W. Tucker
}

The principal result of this paper is the determination of every graph that can be covered by a complete graph. It is shown that for every odd divisor $d$ of the number $n$ of vertices of a complete graph $K_{n}$, there is a unique graph with $n / d$ vertices covered by $K_{n}$, and that there are no other graphs covered by $K_{n}$. This determination is applied to an examination of certain aspects of the solution to the Heawood map-coloring problem. In particular, combinatorial arguments of the solution are set in a topological framework of branched covering spaces.

1. Terminology and one-vertex quotients. A graph is a finite 1-dimensional cell complex, that is, it may have multiple adjacencies and self-adjacencies. (F. Harary [7] calls this a "pseudograph".) Connected graphs are the main concern here.

The complete graph $K_{n}$ is the graph with $n$ vertices and an edge between any pair of vertices. The $d$-fold complete graph on $n$ vertices is the graph with $n$ vertices and exactly $d$ edges between each pair of vertices.

A quotient of a graph $K$ is a graph $K^{\prime}$ such that there exists a regular covering projection $f: K \rightarrow K^{\prime}$. Such a graph $K^{\prime}$ is the quotient of $K$ by the automorphism group of covering translations, but most of the interest here is in finding quotients, not in the different possible projections $K \rightarrow K^{\prime}$ or the associated automorphism groups.

The graph with one vertex and $n$ edges is called a bouquet of $n$ circles. Proposition 1 indicates that every bouquet of circles is a quotient of a complete graph.

Proposition 1. The complete graph $K_{2 n+1}$ covers the bouquet of $n$ circles.

Proof. Label the vertices of $K_{2 n+1}$ by $0, \cdots, 2 n$ modulo $2 n+1$ and the circles of the bouquet by $1, \cdots, n$. For $i=0, \cdots, 2 n$ and $j=1, \cdots, n$, an edge between vertices $i$ and $i+j$ modulo $2 n+1$ projects onto circle $j$.

The complete graph $K_{2 n}$ on evenly many vertices has $n(2 n-1)$ edges, so one might hope it is an $n$-fold cover of some graph with two vertices and $2 n-1$ edges. Proposition 2 indicates that this hope is not realized when the number $n$ is even. 
Proposition 2. Let $d$ be an even divisor of $n$. Then the complete graph $K_{n}$ has no d-fold quotients.

Proof. Suppose that $f: K_{n} \rightarrow K$ is a $d$-fold covering and that $v$ is a vertex of $K$. The edges adjoining the $d$ vertices of the fiber $f^{-1}(v)$ over $v$ form a complete graph, which is the preimage of a set of loops based at $v$. Since the covering is $d$-fold, each $v$-based loop lifts to $d$ edges in that complete graph, contradicting the fact that the even number $d$ does not divide the number $d(d-1) / 2$ of edges in that complete graph.

CoROLlaRY. A complete graph on a number of vertices which is a power of two has no nontrivial quotients.

It is proved in the next section that a complete graph $K_{n}$ has a unique $d$-fold quotient for every odd divisor $d$ of $n$ and no other quotients. While an elementary proof is feasible, the details required to establish existence are automatically provided by the voltage graph construction, which is needed for the remainder of the paper.

In addition to satisfying the usual topological criteria (e.g., see Chapter 5 of W. S. Massey [11]), the covering maps in the paper are assumed to be cellular, that is, each cell of the covering space lies over a cell of the base, necessarily of the same dimension.

2. Reduced voltage graphs. Voltage graphs are introduced by J. L. Gross [2] to assist in the construction of graph imbeddings in surfaces, in particular, to help compute the genus of graphs. The theory of voltage graphs is dual to that of current graphs, originated in combinatorial form by W. Gustin [6], exploited by G. Ringel and J. W. T. Youngs [17] and their colleagues in the solution of the Heawood map-coloring problem, and recently developed into full topological generality by Gross and S. R. Alpert [3, 4]. This section provides further illustration of their advantage over current graphs in certain applications.

A reduced voltage graph is a pair $(K, \beta)$ consisting of a graph $K$ and a set function $\beta$ from the oriented edges of $K$ to a group $G$ (usually finite) such that the value of $\beta$ on an edge is the inverse in $G$ of its value on the reverse edge (i.e., the oriented edge with the same endpoints but opposite direction). The values of $\beta$ are called voltages.

To any reduced voltage graph $(K, \beta)$ there is associated a derived graph $K^{\beta}$ whose vertex set is the cartesian product $V \times G$ of the set $V$ of vertices of $K$ and the voltage group $G$. For every edge $k$ between vertices $u$ and $v$ of the graph $K$ and every $g \in G$, there is an edge 
in $K^{\beta}$ between the vertices $(u, g)$ and $(v, b g)$, where $b$ is the voltage from $u$ to $v$ on $k$. If $k$ is regarded as carrying voltage $b^{-1}$ from $v$ to $u$, then that same edge in $K^{\beta}$ is generated by the element $b g \in G$, that is, it lies between the vertices $(v, b g)$ and $\left(u, b^{-1}(b g)\right)=(u, g)$.

One advantage of voltage graphs over current graphs apparent in the above definition is that the derived graph $K^{\beta}$ is independent of an imbedding of $K$ in a surface.

THEOREM 1. Let $(K, \beta)$ be a reduced voltage graph with voltages in a group $G$. Then the derived graph $K^{\beta}$ is a (possibly disconnected) $|G|$-fold covering of $K$.

Proof. The graph $K^{\beta}$ as constructed above admits a graph morphism onto $K$ which carries a vertex $(v, g)$ onto its first coordinate and an edge between $(u, g)$ and $(v, b g)$ onto the edge $k$ between $u$ and $v$ that contributed to its generation. The voltage group $G$ acts as an automorphism group on $K^{\beta}$ by what is essentially right translation, that is, for all $h \in G$

$$
(v, g) \cdot h \longrightarrow(v, g h)
$$

and

$$
[(u, g),(v, b g)] \cdot h \longrightarrow[(u, g h),(v, b g h)] .
$$

The graph morphism $K^{\beta} \rightarrow K$ realizes the quotient of $K^{\beta}$ under the automorphism group $G$, so it is a regular covering (on every component of $K^{\beta}$ ). One observes that the transitivity of the action assures the isomorphism of components of $K^{\beta}$.

The above proof of Theorem 1 is abstracted from the proof of Theorem 1 of Gross and Alpert [4], where it was necessary to be concerned with extending the graph covering projection to the imbedding surfaces. It is now applied to completing the classification of quotients of complete graphs.

THEOREM 2. A complete graph $K_{n}$ has a unique d-fold quotient for every odd divisor $d$ of $n$ and no other quotients. Such a quotient is obtained by amalgamating a bouquet of $(d-1) / 2$ circles to every vertex of a d-fold complete graph on $n / d$ vertices.

Proof. Suppose that $K_{n}$ has a $d$-fold quotient $K$, for some odd divisor $d$ of $n$. Then the edges adjoining the $d$ vertices in the fiber over any vertex of $K$ form a complete graph, which necessarily projects onto a bouquet of $(d-1) / 2$ circles. The $d^{2}$ edges adjoining the vertices of two distinct fibers form a complete bipartite graph 
that projects onto $d$ edges between the two base vertices in $K$. This establishes the unique isomorphism type of a $d$-fold quotient. By Proposition 2, there are no even quotients. What remains to be proved is the existence of all odd quotients, and this is where reduced voltage graphs are useful.

For any odd divisor $d$ of $n$, let $L_{d}$ be a graph obtained by amalgamating a bouquet of $(d-1) / 2$ circles to every vertex of a $d$ fold complete graph on $n / d$ vertices. At every vertex of $L_{d}$, give each of the circles a preferred direction and assign the voltages 1 , $\cdots,(d-1) / 2$ modulo $d$ to the circles with preferred directions and the inverse voltages $d-1, d-2, \cdots,(d+1) / 2$ to their opposites. The $d$ edges in $L_{d}$ running from any vertex $v$ to any other vertex $u$ are assigned the voltages $0, \cdots, d-1$ modulo $d$ (assuring that their respective opposites are assigned the voltages $0, d-1, d-2, \cdots, 1$ modulo $d$ ). The construction of the derived graph $L_{d}^{\beta}$ corresponding to the reduced voltage graph $\left(L_{d}, \beta\right)$ assures that it is a complete graph on $n$ vertices.

Precisely, the derived graph $L_{d}^{\beta}$ has $n(n-1) / 2$ edges. There are no self-adjacencies, because no identity voltage is assigned to a selfadjacency in $L_{d}$ and no multiple adjacencies since no two distinct edges in $L_{d}$ between the same pair of vertices are assigned the same voltages. By the "pigeonhole principle", there must be exactly one edge between each pair of vertices in the derived graph. This completes the proof.

3. Branched coverings. In preparation for consideration of the solution to the Heawood map-coloring problem, this section defines voltage graphs in full generality and gives the main theorem. The exposition here differs from that of Gross [2] in that it avoids "rotation systems", thereby permitting, incidentally, an extension of the theory to nonorientable graph imbeddings. An understanding of what follows depends on comprehension of the notion of a branched covering.

A cellular map $f: X \rightarrow Y$ between two cell complexes is called a branched covering if $X$ and $Y$ have subdivisions $X^{\prime}$ and $Y^{\prime}$ preserving the cellularity of $f$ such that the restriction of $f$ to the complement in $X^{\prime}$ of a subcomplex of codimension two is topologically a covering.

The subdividing permitted by this combinatorial definition of branched covering is of critical importance here since, in what follows, that branching set of the covering space is a discrete set of points, each lying in the interior of a polygon of, say, $n$ sides, whose image is a polygon of $n / d$ sides for some divisor $d$ of $n$. The canonical model of such a branched covering action is the map $r e^{i \theta} \rightarrow r e^{i d \theta}$ on the complex plane, which has a branch point of degree $d$ at the origin. A voltage graph is a triple $(K, \beta, c: K \rightarrow M)$ such that the pair 
$(K, \beta)$ is a reduced voltage graph and $c: K \rightarrow M$ is an imbedding of $K$ as the 1 -skeleton of a closed polyhedral surface $M$.

The product of the voltages on the edges comprising the boundary of a face of the imbedding $c: K \rightarrow M$ is called an excess voltage. Such a product is computed in sequential order, and it may differ by conjugacy in the voltage group from another such product, computed from a different starting edge. The order of the excess voltage in the voltage group is unique. The Kirchoff voltage law (abbr. KVL) is said to hold on any face where the excess voltage is the identity.

By construction of the derived graph $K^{\beta}$, each face boundary (or, more generally, any cycle) in $K$ with $n$ edges lifts to a set of cycles in $K^{\beta}$ each of whose number of edges is the product of $n$ and the order of its excess voltage.

A derived surface $M^{\beta}$ and a derived imbedding $c^{\beta}: K^{\beta} \rightarrow M^{\beta}$ are obtained by identifying each component of a lifted face boundary with the sides of a 2-cell (unique to that component). The covering $K^{\beta} \rightarrow K$ is readily extended to a surface map $M^{\beta} \rightarrow M$. Details of this construction may be read (from a current graph viewpoint) in Gross and Alpert [4]. Gross [2] uses Theorem 2 of [4] to prove the following.

THEOREM 3. Let the voltage graph $(K, \beta, c: K \rightarrow M)$ with voltages in a group $G$ have faces $f_{1}, \cdots, f_{q}$ with $s_{1}, \cdots, s_{q}$ sides and carrying excess voltages of orders $e_{1}, \cdots, e_{q}$ respectively. Then the derived imbedding $c^{\beta}: K^{\beta} \rightarrow M^{\beta}$ is a branched covering of the imbebbing $c: K \rightarrow$ $M$. For $i=1, \cdots, q$ there are $|G| \mid e_{i}$ faces lying over face $f_{i}$, each with $e_{i} s_{i}$ sides and (for $e_{i} \neq 1$ ) a branch point of degree $e_{i}$ in its interior.

4. Revisiting the Heawood map-coloring problem. A coloring of a closed orientable surface $T_{h}$ with $h$ handles is an assignment of colors to the faces of a polyhedral decomposition such that no two adjacent faces are assigned the same color. The chromatic number $\operatorname{chr}\left(T_{h}\right)$ is the minimum number such that any polyhedral decomposition of $T_{h}$ has a coloring with that number of colors.

In 1890, P. J. Heawood proved that

$$
\operatorname{chr}\left(T_{h}\right) \leqq\left\lfloor\frac{7+\sqrt{1+48 h}}{2}\right\rfloor \quad h \geqq 1
$$

where $\lfloor x\rfloor$ denotes the greatest integer (or "floor") in a real number $x$. The right side of the above inequality is denoted $H(h)$ and called the Heawood number of $T_{h}$. Heawood conjectured that his upper bound was an equality.

One observes that to prove the Heawood conjecture, it is sufficient 
to imbed the complete graph on $H(h)$ vertices in the surface $T_{h}$, for the dual imbedding would require $H(h)$ colors. In other words, it is sufficient to prove that the genus of the graph $K_{H(h)}$ is less than or equal to $h$.

Ringel, Youngs, and their colleagues solved the problem by proving that

$$
\text { genus }\left(K_{n}\right)=\left\lceil\frac{(n-3)(n-4)}{12}\right\rceil n \geqq 3
$$

where $\lceil x\rceil$ denotes the least integer ("ceiling") greater than or equal to a real number $x$. This equation is of ten called the Ringel-Youngs equation. Its right side is denoted $I(n)$. Routine manipulation of the expression $I(H(h))$ establishes that genus $\left(K_{H(h)}\right)$ is less than or equal to $h$.

Heawood's conjecture appears in the same paper in which he disclosed the flaw in the purported proof by A. B. Kempe [10] of the four color conjecture, which, one notices, is obtained by substituting zero for $h$ in Heawood's conjecture.

The proof of the Ringel-Youngs equation analyzes each residue class of $n$ modulo 12 separately, for reasons that are soon apparent in a serious investigation of the problem, but that might yet be overcome, some would hope. The full proof occupies about 300 published pages. The difficulty of proof varies widely from one residue class to another.

The Euler formula $V-E+F=2-2 g$ and the edge-face inequality $2 E \geqq 3 F$ lead to the complete graph inequality

$$
\text { genus }\left(K_{n}\right) \geqq I(n) \quad n \geqq 3 \text {. }
$$

Thus, the Ringel-Youngs equation can be affirmed by constructing an imbedding of the complete graph $K_{n}$ in the surface $T_{I(n)}$. Such an imbedding maximizes the number of faces, subject to the above inequality. Maximizing the number of faces is accomplished, in turn, by making as many of them triangles as is possible.

For $n$ congruent to $0,3,4$, or 7 modulo 12 the quantity in brackets in the Ringel-Youngs equation is an integer, which is equivalent to the statement that a complete graph on a number of vertices in any of these residue classes has a triangular imbedding in an orientable surface. No other residue class has a triangular imbedding, by the complete graph inequality.

5. Residue class 7. The easiest case to understand is residue class 7, originally solved by Ringel [16] and later rewritten by Youngs [24] in a fashion consistent with the published solutions of other cases. 
The vertex, edge, and face numbers for a triangular imbedding of a complete graph $K_{12 s+7}, s=0,1, \cdots$, are $12 s+7$ vertices, $(12 s+$ $7)(6 s+3)$ edges, and $(12 s+7)(4 s+2)$ faces. They are all divisible by $12 s+7$, suggesting the possibility that such an imbedding is a $(12 s+$ 7)-fold unbranched cover of something, necessarily a triangular imbedding whose vertex, edge, and face numbers are $1,6 s+3$, and $4 s+2$, that is, a bouquet of $6 s+3$ circles in a surface of genus $s+1$.

A common factor in the vertex, edge, and face numbers is no immediate assurance of a quotient imbedding, or even of the existence of an imbedding whose cell numbers make it a candidate for a quotient. In this case, however, ther are many candidates, that is, triangular imbeddings of bouquets of $6 s+3$ circles. In general, a candidate for a quotient is not unique.

Figure 1 illustrates an imbedding of the bouquet of 9 circles that is a quotient of a triangular imbedding of $K_{19}$, thereby verifying the Ringel-Youngs equation for $n=19$. Two edges of the octogon boundary are identified if they carry the same voltage in the cyclic group $Z_{19}$.

The bouquet of nine circles is a quotient for many different 19 vertex graphs, only one of which has no multiple edges. What assures that the derived graph here is $K_{19}$ is that it has 9.19 edges and there are no self-adjacencies or multiple adjacencies. (No edge has identity

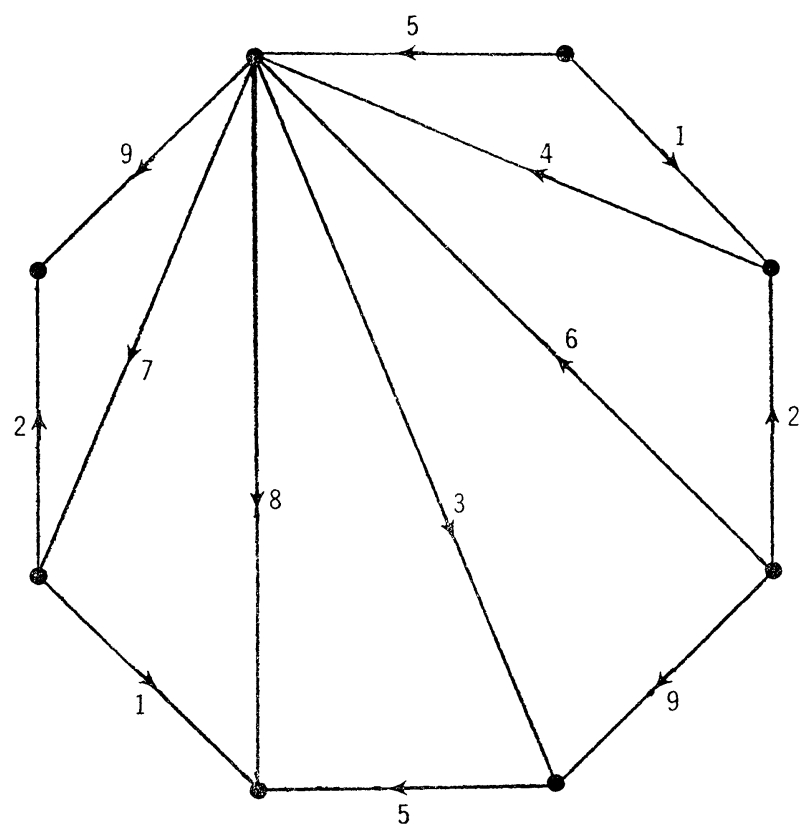

FIgURE 1. A quotient for a minimal imbedding of the complete graph $K_{19}$, with voltages in $Z_{19}$. 
voltage and no two distinct oriented edges have the same voltage.)

What assures that the derived imbedding is triangular is that KVL holds on every face of the quotient imbedding.

If the Gustin ladder-like nomogram for $K_{19}$ with its coil-derived currents (see Youngs [23]) is topologically interpreted as a current graph in the sense of Gross and Alpert [4], then that current graph is precisely the dual of the voltage graph illustrated here in Figure 1. In fact, every current graph used in the published solution for residue class 7 modulo 12 has as its dual a voltage graph resembling the one in Figure 1 in the sense that the imbedding looks like a scallop shell.

6. Residue classes 3,4, and 0 . This section completes discussion of the complete graphs that admit triangular imbeddings.

A triangular imbedding of $K_{12 s+3}$ must have $12 s+3$ vertices, $(6 s+1)(12 s+3)$ edges, and $(6 s+1)(8 s+2)$ faces, for $s=0,1, \cdots$. The largest common factor of these cell numbers is $4 s+1$, corresponding to a quotient imbedding of a graph obtained by amalgamating a bouquet of $2 s$ circles to each vertex of a $(4 s+1)$-fold complete graph on three vertices and to an unbranched covering.

The current group employed by Youngs [23] in his unifying version of the solution, originally achieved for residue class 3 by Ringel [16], is not $Z_{4 s+1}$ but $Z_{12 s+3}$. The explanation is that the covering space corresponding to Youngs's nomogram and current distribution has three mutually isomorphic components. Alpert and Gross [1] prove that the number of components (always mutually isomorphic) equals the index in the current group of the isotropy group for any fixed component.

Unfortunately, a quotient imbedding for residue class 3 is not so easily drawn as in residue class 7 , so none is illustrated here.

The cell numbers for a triangular imbedding of $K_{12 s+4}$ are $12_{s}+$ $4,(6 s+2)(12 s+3)$, and $(6 s+2)(8 s+2)$ for $s=0,1, \cdots$. Theorem 2 prohibits use of $6 s+2$, the largest common factor, seemingly leaving the task of constructing a $(3 s+1)$-fold cover. But when $s$ is odd, even

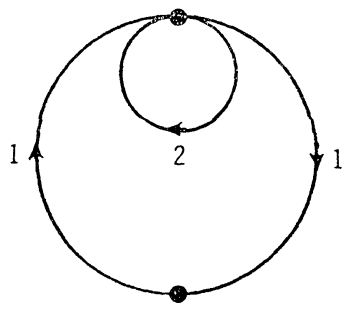

Frgure 2. A quotient graph with one vertex and two edges, imbedded in the projective plane, with voltages in $Z_{4}$, leading to a minimal imbedding of the complete graph $K_{4}$ in the sphere $T_{0}$. 
the factor $3 s+1$ is impermissible.

A different tactic, however, is illustrated by Figure 2. From the voltage graph shown, one derives a nearly triangular imbedding of a graph which is nearly $K_{4}$.

By construction, the derived graph for Figure 2 has $8(=2 \cdot 4)$ edges. It is $K_{4}$ plus two redundant edges. The voltage 2 on the central loop in Figure 2 is of order 2 in the group $Z_{4}$, so the monogon it encloses lifts to two digons, by Theorem 3. Since KVL holds on the other face, the remaining faces in the derived imbedding are triangles. Excising the digons and reclosing the surface by identification of multiple edges eliminates all eight redundancies and yields a triangular imbedding $K_{4} \rightarrow T_{0}$.

Figure 2 is apparently the first example of a nonorientable quotient graph imbedding used to derive an orientable imbedding (by either voltages or currents). Unfortunately, there seems to be no obvious generalization to a new solution for all of residue class 4 .

What C. M. Terry, L. R. Welch, and Youngs [14] did in their solution to cases 4 was to employ the above tactic in the combinatorial guise of "singular arcs", invented by Gustin [6], and interpreted topologically by Youngs [22] (or see Gross and Alpert [4]). The problem solvers added $3(6 s+2)$ redundant edges and digons, yielding a derived imbedding with $12 s+4$ vertices, $(12 s+4)(6 s+3)$ edges, $(12 s+4)(4 s+$ 1) triangles, and $18 s+6$ digons. They employed a $(12 s+4)$-fold branched cover, obtained from currents in $Z_{2} \times Z_{6 s+2}$. Thus, the dual of their current graph (interpreted topologically) has 1 vertex, $6 s+$ 3 edges, $4 s+1$ triangles on which KVL holds, and 3 monogons whose excess voltage has order 2 .

Residue class 0 presents unusual difficulty, since a number of the form $12 s$ might have no large odd factors. Terry, Welch, and Youngs [13] solved this case also, once again using singular arcs, and introducing the use of a nonabelian current group.

A topological interpretation of their method is that the problem solvers added $6 s\left(2^{k}-1\right)$ redundant edges and digons to the desired imbedding, thereby allowing it to be obtained via a $12 s$-fold branched covering. The number $2^{k}$ is the largest power of two that divides $12 s$.

7. Other residue classes. If the number $n$ is not congruent to $0,3,4$, or 7 modulo 12 , then there is no triangular imbedding of the complete graph $K_{n}$. The strategy for these "irregular" cases is to discard one or more edges of the complete graph, to then triangularly imbed the resulting graph, and to finally restore the deleted edges on one or more handles, thereby obtaining an imbedding verifying the Ringel-Youngs equation. 
The first residue class for which the problem was solved was residue class 5, by Ringel [15]. Of the irregular cases, it is the easiest to describe.

Suppose there were an imbedding of the complete graph $K_{128+3}$, $s=0,1, \cdots$, in the surface $T_{I(12 s+5)-1}$ such that every face was 3-sided except for two $(12 s+3)$-gons, both of whose boundaries contained each vertex exactly once. Then two additional vertices might be inserted as the "barycenters" of the $(12 s+3)$-gons and adjoined to each boundary vertex, thereby yielding an imbedding of $K_{128+5}-K_{2}$ (i.e., the edge-complement of $K_{2}$ in $\left.K_{12 s+5}\right)$ in $T_{I(12 s+5)-1}$. By attaching a handle from the interior of a triangle incident on one of the two new vertices to the interior of a triangle incident on the other, and by then adjoining the two vertices over that handle, one could obtain the desired imbedding $K_{12 s+5} \rightarrow T_{I(12 s+5)}$.

The current graph used to actually construct the imbedding of $K_{12 s+3}$ in $T_{I(12 s+5)-1}$ resembles the one used for residue class 3 .

Another early residue class to yield was residue class 10, solved by Ringel [16]. By an extension of the combinatorial devises used for the triangular imbedding of $K_{12 s+7}$, it is possible to imbed $K_{12 s+7}$ in $T_{I(12 s+10)-1}$ so that every face is 3-sided except for three complete $(12 s+7)$-gons. Inserting additional vertices and adjoining edges as in case 5 yields a triangular imbedding of $K_{12 s+10}-K_{3}$ in $T_{I(12 s+10)-1}$. Ringel developed an ingenious way of adding a single handle (see Section 1 of Youngs [23]) so that the three mutually nonadjacent vertices could be made mutually adjacent.

The relation of residue class 6 to class 3 is like that of class 10 to class 7. The solution for residue classes 1 and 9 employ redundant edges and digons. The current distributions for classes 6 and 9 are especially difficult to construct.

The solution of Ringel and Youngs [19] for residue class 11 is obtained by imbedding $K_{12 s+6}$ in $T_{I(12 s+11)-2}$ with five $(12 s+6)$-gons, augmenting to obtain a triangular imbedding of $K_{12 s+11}-K_{5}$, and then adding two handles and some adjacencies to produce $K_{12 s+11}$ in $T_{I(12 s+11)}$. Addition of the ten needed adjacencies is not directly accomplished. There are several intermediate steps involving adjacency modifications, where a "modification" means replacing the existing diagonal of a quadrilateral by the other diagonal.

The novelty in the solution of Ringel and Youngs [21] for residue class 8 is that it is obtained via an imbedding of $K_{12 s+6}$ in $T_{I(12 s+6)-1}$ whose faces are triangular except for one $(12 s+6)$-gon and two $(6 s+3)$-gons. After the usual augmenting procedure, the vertices inserted at the barycenters of the $(6 s+3)$-gons are amalgamated and the vertex at the barycenter of the $(12 s+6)$-gon is adjoined to the amalgamated vertex. The amalgamation and adjoining operation are 
jointly achieved with the addition of only one handle and some adjacency modifications.

The solutions of Ringel and Youngs [20] for residue class 2 involves amalgamating a vertex as in case 8 , adding six handles, and performing numerous adjacency modifications. For $s$ odd, Ringel and Youngs [18] also obtained a triangular imbedding of $K_{12 s+2}-K_{2}$ in $T_{I(12 s+2)-1}$. Mahendra Jungerman [25] has found a triangular imbedding of $K_{12 s+2}-K_{2}$ for all $s=0,1, \cdots$ and also a shorter solution for residue class 9 .

This revisit would not be complete without crediting L. Heffter [9] for inventing a valuable method for describing graph imbeddings and for constructing some special imbeddings of complete graphs $K_{n}$, $n \leqq 12$ or without mentioning the imbeddings constructed by J. Mayer [12] for complete graphs $K_{n}(n=18,22,23,30,35,47,59)$ which resisted the methods described here. Also, Ringel and Youngs [17] credit R. K. Guy for contributions to the solution and also credit Gustin for collaboration on cases 1 and 9 and an independent solution for residue class 4 .

Finally, another paper of the present authors [5] proves that every graph covering, regular or irregular, results from some voltage assignment on the given graph.

\section{REFERENCES}

1. S. R. Alpert and J. L. Gross, Components of branched coverings of current graphs, in preparation.

2. J. L. Gross, Voltage graphs, Discrete Math., in press.

3. J. L. Gross and S. R. Alpert, Branched coverings of graph imbeddings, Bull. Amer. Math. Soc., 79 (1973), 942-945.

4. - The topological theory of current graphs, J. Combinatorial Theory, to appear.

5. J. L. Gross and T. W. Tucker, Generating all graph coverings by voltage distributions, in preparation.

6. W. Gustin, Orientable embeddings of Cayley graphs, Bull. Amer. Math. Soc., 69 (1963), 272-275.

7. F. Harary, Graph Theory, Addison-Wesley, Reading, Massachusetts, 1969.

8. P. J. Heawood, Map color theorem, Quart. J. Math., 24 (1890), 332-338.

9. L. Heffter, Über das Problem der Nachbargebiete, Math, Ann., 38 (1891), 477-508.

10. A. B. Kempe, On the geographical problem of four colors, Amer. J. Math., 2 (1879), 193-204.

11. W. S. Massey, Algebraic Topology: An Introduction, Harcourt, Brace \& World, New York, 1967.

12. J. Mayer, Le problème des régions voisines sur les surfaces closes orientables, J. Combinatorial Theory, 6 (1969), 177-195.

13. C. M. Terry, L. R. Welch, and J. W. T. Youngs, The genus of $K_{12 s}$, J. Combinatorial Theory, 2 (1967), 43-60.

14. - Solution of the Heawood map-coloring problem-case 4, J. Combinatorial Theory, 8 (1970), 170-174. 
15. G. Ringel, Bestimmung der Maximalzahl der Nachbargebiete auf nichtorientierbaren Flächen, Math. Ann., 127 (1954), 181-214.

16. - Über das Problem der Nachbargebiete auf orientierbaren Flächen, Abh. Math. Sem. Univ. Hamburg, 25 (1961), 105-127.

17. G. Ringel and J. W. T. Youngs, Solution of the Heawood map-coloring problem, Proc. Nat. Acad. Sci. U. S. A., 60 (1968), 438-445.

18. - Lösung des Problems der Nachbargebiete auf orientierbaren Flächen, Archiv der Mathematik, 20 (1969), 190-201.

19. - Solution of the Heawood map-coloring problem-case 11, J. Combinatorial Theory, 7 (1969), 71-93.

20. - Solution of the Heawood map-coloring problem-case 2, J. Combinatorial Theory, 7 (1969), 342-352.

21. Solution of the Heawood map-coloring problem-case 8, J. Combinatorial, Theory, 7 (1969), 353-363.

22. J. W. T. Youngs, The Heawood Map-coloring Conjecture, Graph Theory and Theoretical Physics, Academic Press, London, 1967, pp. 313-354.

23. - Solution of the Heawood map-coloring problem-cases 3, 5, 6 and 9, J. Combinatorial Theory, 8 (1970), 175-219.

24. J. W. T. Youngs, Solution of the Heawood map-coloring problem-cases, 1, 7, and 10, J. Combinatorial Theory, 8 (1970), 220-231.

25. M. Jungerman, Ph. D. dissertation, University of California at Santa Cruz, 1974.

Received May 2, 1974. The first author is an Alfred P. Sloan Fellow. His research is partially supported by National Science Foundation contract GJ34738 at Columbia University. The research of the second author is partially supported by National Science Foundation contract GP34324X at Princeton University. His present address is Colgate University, Hamilton, N. Y. 13346.

Columbia University

AND

Princeton UNIVERSITY 


\section{PACIFIC JOURNAL OF MATHEMATICS}

\section{EDITORS}

RICHARD ARENS (Managing Editor)

University of California

Los Angeles, California 90024

\section{R. A. Beaumont \\ University of Washington \\ Seattle, Washington 98105}

\section{J. DugundJI}

Department of Mathematics

University of Southern California

Los Angeles, California 90007

D. Gilbarg and J. Milgram

Stanford University

Stanford, California 94305

\section{ASSOCIATE EDITORS}
E. F. BECKENBACH
B. H. NeumanN
F. WOLF
K. YOSHIDA

\section{SUPPORTING INSTITUTIONS}

UNIVERSITY OF BRITISH COLUMBIA
CALIFORNIA INSTITUTE OF TECHNOLOGY
UNIVERSITY OF CALIFORNIA
MONTANA STATE UNIVERSITY
UNIVERSITY OF NEVADA
NEW MEXICO STATE UNIVERSITY
OREGON STATE UNIVERSITY
UNIVERSITY OF OREGON
OSAKA UNIVERSITY

UNIVERSITY OF BRITISH COLUMBIA

UNIVERSITY OF CALIFORNIA

MONTANA STATE UNIVERSITY

NEW MEXICO STATE UNIVERSITY

OREGON STATE UNIVERSITY

OSAKA UNIVERSITY

\author{
UNIVERSITY OF SOUTHERN CALIFORNIA \\ STANFORD UNIVERSITY \\ UNIVERSITY OF TOKYO \\ UNIVERSITY OF UTAH \\ WASHINGTON STATE UNIVERSITY \\ UNIVERSITY OF WASHINGTON \\ AMERICAN MATHEMATICAL SOCIETY \\ NAVAL WEAPONS CENTER
}

The Supporting Institutions listed above contribute to the cost of publication of this Journal, but they are not owners or publishers and have no responsibility for its content or policies.

Mathematical papers intended for publication in the Pacific Journal of Mathematics should be in typed form or offset-reproduced, (not dittoed), double spaced with large margins. Underline Greek letters in red, German in green, and script in blue. The first paragraph or two must be capable of being used separately as a synopsis of the entire paper. Items of the bibliography should not be cited there unless absolutely necessary, in which case they must be identified by author and Journal, rather than by item number. Manuscripts, in triplicate, may be sent to any one of the editors. Please classify according to the scheme of Math. Reviews, Index to Vol. 39. All other communications should be addressed to the managing editor, or Elaine Barth, University of California, Los Angeles, California, 90024.

The Pacific Journal of Mathematics expects the author's institution to pay page charges, and reserves the right to delay publication for nonpayment of charges in case of financial emergency.

100 reprints are provided free for each article, only if page charges have been substantially paid. Additional copies may be obtained at cost in multiples of 50 .

The Pacific Journal of Mathematics is issued monthly as of January 1966. Regular subscription rate: $\$ 72.00$ a year (6 Vols., 12 issues). Special rate: $\$ 36.00$ a year to individual members of supporting institutions.

Subscriptions, orders for back numbers, and changes of address should be sent to Pacific Journal of Mathematics, 103 Highland Boulevard, Berkeley, California, 94708.

PUBLISHED BY PACIFIC JOURNAL OF MATHEMATICS, A NON-PROFIT CORPORATION

Printed at Kokusai Bunken Insatsusha (International Academic Printing Co., Ltd.), 270, 3-chome Totsuka-cho, Shinjuku-ku, Tokyo 160, Japan.

Copyright (C) 1973 by Pacific Journal of Mathematics Manufactured and first issued in Japan 


\section{Pacific Journal of Mathematics \\ Vol. 55, No. $2 \quad$ October, 1974}

Walter Allegretto, On the equivalence of two types of oscillation for elliptic

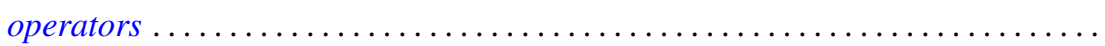

Edward Arthur Bertram, A density theorem on the number of conjugacy classes in

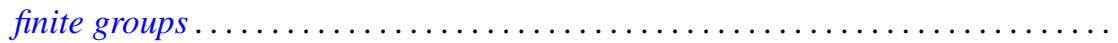

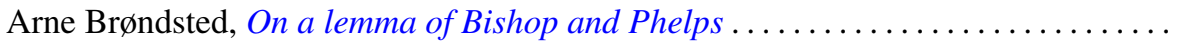

Jacob Burbea, Total positivity and reproducing kernels ..................

Ed Dubinsky, Linear Pincherle sequences . . . . . . . . . . . . . . . . . .

Benny Dan Evans, Cyclic amalgamations of residually finite groups .............

361

Barry J. Gardner and Patrick Noble Stewart, A "going down" theorem for certain

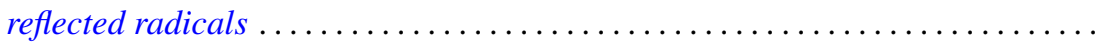

381

Jonathan Light Gross and Thomas William Tucker, Quotients of complete graphs:

revisiting the Heawood map-coloring problem ....................

Sav Roman Harasymiv, Groups of matrices acting on distribution spaces .........

Robert Winship Heath and David John Lutzer, Dugundji extension theorems for

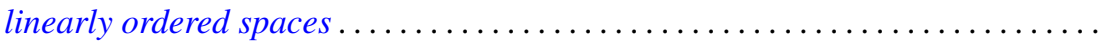

Chung-Wu Ho, Deforming p. l. homeomorphisms on a convex polygonal

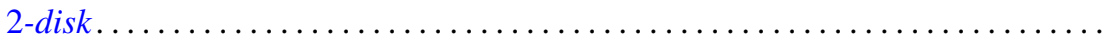

Richard Earl Hodel, Metrizability of topological spaces .................

Wilfried Imrich and Mark E. Watkins, On graphical regular representations of

cyclic extensions of groups .......................... 461

Jozef Krasinkiewicz, Remark on mappings not raising dimension of curves ..... . 479

Melven Robert Krom, Infinite games and special Baire space extensions . . . . . . 483

S. Leela, Stability of measure differential equations . . . . . . . . . . . . . . . . 489

M. H. Lim, Linear transformations on symmetric spaces . . . . . . . . . . . . . . . 499

Teng-Sun Liu, Arnoud C. M. van Rooij and Ju-Kwei Wang, On some group algebra modules related to Wiener's algebra $M_{1} \ldots \ldots \ldots \ldots \ldots \ldots \ldots \ldots \ldots \ldots \ldots$

Dale Wayne Myers, The back-and-forth isomorphism construction ............ 521

Donovan Harold Van Osdol, Extensions of sheaves of commutative algebras by

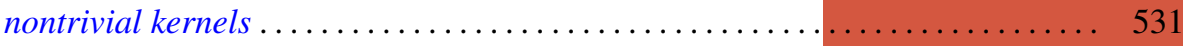

Alan Rahilly, Generalized Hall planes of even order ................... 543

Joylyn Newberry Reed, On completeness and semicompleteness of first countable

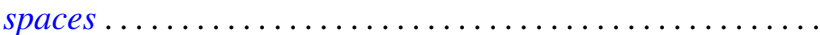

Alan Schwartz, Generalized convolutions and positive definite functions associated

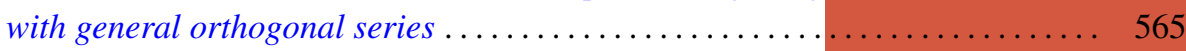

Thomas Jerome Scott, Monotonic permutations of chains . . . . . . . . . . . 583

Eivind Stensholt, An application of Steinberg's construction of twisted groups .... 595

Yasuji Takeuchi, On strongly radicial extensions . . . . ................. 619

William P. Ziemer, Some remarks on harmonic measure in space . . . . . . . . . . 629

John Grant, Corrections to: “Automorphisms definable by formulas” . . . . . . . . 639

Peter Michael Rosenthal, Corrections to: "On an inversion for the general

Mehler-Fock transform pair" ......................... 640

Carl Clifton Faith, Corrections to: "When are proper cyclics injective” . . . . . . 640 